\title{
SCATTERING THEORY FOR HYPERBOLIC SYSTEMS WITH COEFFICIENTS OF GEVREY TYPE ${ }^{(1)}$
}

\author{
BY
}

\author{
WILLIAM L. GOODHUE
}

ABSTRACT. Using the techniques developed by P. D. Lax and R. S. Phillips, qualitative results on the location of the poles of the scattering matrix for symmetric, hyperbolic systems are obtained. The restrictions placed on the system are that the coefficient matrices be of Gevrey type and that the bicharacteristic rays tend to infinity.

Introduction. In a series of papers and a mongraph, P. D. Lax and R. S. Phillips [5a, b, c] have developed a scattering theory for symmetric hyperbolic systems of partial differential equations. Of primary concern in all scattering work is the location of the poles of the scattering matrix, and in particular, how their location depends on the scatterer. Using the techniques developed by Lax and Phillips, it will be shown that if the bicharacteristic rays tend to $\infty$, then for coefficient matrices of Gevrey type, the poles of the scattering matrix are bounded away from the real axis, with the possible exception of a finite segment centered at the origin. A similar result holds for the acoustic equation with a potential of Gevrey type.

The author wishes to express his gratitude to Professor P. D. Lax for his guidance and advice during the period in which this paper was researched.

I. Review of scattering theory for symmetric hyperbolic systems; statement of results. The "perturbed" system under consideration is the following one:

$$
\begin{aligned}
\frac{\partial}{\partial t} u(x, t) & =\sum_{j=1}^{k} A^{j}(x) \frac{\partial}{\partial x_{j}} u(x, t)+B(x) u(x, t) \equiv G u, \\
u(x, 0) & =f(x),
\end{aligned}
$$

where $u(x, t)$ is an $n$-vector depending on $x=\left(x_{1}, \cdots, x_{k}\right)$ and $t$. The $n \times n$ matrices $A^{j}(x), B(x)$ are assumed to satisfy the conditions below:

Received by the editors July 24, 1972. $81 \mathrm{~A} 48$.

AMS (MOS) subject classifications (1970). Primary 47A40, 35P25, 35L45; Secondary

Key words and phrases. Scattering matrix poles, hyperbolic system s, spectrum of infinitesimal generator.

(1) The results of this report were contained in the author's doctoral dissertation, written under Professor P. D. Lax at New York University. 
(A1) The coefficients of $\left\{A^{j}(x)\right\}_{j=1}^{k}$ and $B(x)$ are infinitely differentiable, and moreover, if $\left\{A_{0}^{j}\right\}_{j=1}^{k}$ represents a set of constant coefficient matrices, the matrices $A^{j}(x)-A_{0}^{j}$ and $B(x)$ have support contained within the ball $|x|=\rho$.

(A2) The matrices $A^{j}(x)$ are Hermitian and $B(x)+B^{*}(x)=\sum_{j=1}^{k} \partial A^{j}(x) / \partial x_{j}$, so that the operator $G$, defined on the Hilbert space $\mathcal{H}=\left[L_{2}\left(R^{k}\right)\right]^{n}$, is skew-symmetric.

(A3) The propagation speeds for the system (1.1) are all nonzero and distinct; i.e., for any nonzero $k$-vector $\xi$, the matrix $\sum_{j=1}^{k} \xi_{j} A^{j}(x)$ has distinct, nonzero eigenvalues $\left\{\tau^{\lambda}(x, \xi)\right\}_{\lambda=1}^{n}$ which may be ordered so that

$$
\tau^{1}(x, \xi)>\cdots>\tau^{n / 2}(x, \xi)>0>\tau^{n / 2+1}(x, \xi)>\cdots>\tau^{n}(x, \xi) .
$$

(A4) The operator $G$ possesses the unique continuation property. In particular, we assume that the complex characteristics of $G$ have multiplicity one.

(A5) The number of space dimensions $k$ is odd and, therefore, the unperturbed system

$$
\begin{aligned}
\frac{\partial}{\partial t} v(x, t) & =\sum_{j=1}^{k} A_{0}^{j} \frac{\partial}{\partial x_{j}} v(x, t) \equiv G_{0} v, \\
v(x, 0) & =f(x)
\end{aligned}
$$

satisfies Huygen's principle.

A classical result [5a] states that there exists a well-defined, one parameter, unitary group of operators $V(t)$, defined on $\mathcal{H}$, such that

$$
V(t): \mathcal{H} \rightarrow \mathcal{H}, \quad f(x) \rightarrow v(x, t) .
$$

Or, in other words, the problem (1.2) is well-posed in the space $\mathcal{H}$. Lax and Phillips [5a, b] have demonstrated that associated with the group $V(t)$ and the space $\mathcal{H}$ are two subspaces of $\mathcal{H}, D_{+}$and $D_{-}$, satisfying

(I) $V(t) D_{+} \subset D_{+}, V(-t) D_{-} \subset D_{-}$, for $t \geq 0$,

(II) $\bigcap_{V(t) D_{+}}=\{0\}=\bigcap \overline{v(-t) D^{-}}$,

(III) $\overline{\bigcup V V(t) D_{+}}=\mathcal{H}=\overline{\bigcup V(-t) D_{-}}$.

The subspaces $D_{+}^{\rho}$ and $D_{-}^{\rho}$ are now defined to be $V(\rho) D_{+}$and $V(-\rho) D_{-}$respectively. It can be shown that the dimension of the null space of $G$ (denoted by $N$ ) is finite and that $\left\{U(-r) D_{+}^{\rho}: \forall r>0\right\}$ is dense in $\mathcal{H}^{\prime}=\mathcal{H} \ominus N$.

The wave operators

$$
W_{ \pm}=\lim _{\substack{t \rightarrow \pm \infty \\ t \rightarrow \pm}} U(-t) V(t)
$$

are defined next, where

$$
U(t): \mathcal{H} \rightarrow \mathcal{H}, \quad f(x) \rightarrow U(x, t)
$$


is the solution operator for the system (1.1), and it follows from the above "density lemma" that (see [5a])

Theorem 1. (i) The wave operators $W_{ \pm}$exist.

(ii) Range $\left(W_{+}\right)=\mathfrak{H}^{\prime}=$ Range $\left(W_{-}\right)$.

(iii) The scattering operator $S=\bar{W}_{+}^{-1} W_{-}$exists and is unitary on $\mathcal{H}$.

To study $U(t)$ more closely, a new operator

$$
Z(t)=P_{+}^{\rho} U(t) P_{-}^{\rho}
$$

is defined, where $P_{ \pm}^{\rho}$ are the orthogonal projections onto $\left(D_{ \pm}^{\rho}\right)^{\perp}$ respectively. The following results are essential in what follows. (See [5a].)

Theorem 2. (1) $Z(t)$ forms a one parameter, strongly cont inuous semigroup on $K^{\rho}=\mathfrak{H}^{\prime} \ominus\left(D_{+}^{\rho} \oplus D_{-}^{\rho}\right)$.

(2) $\|Z(t)\| \leq 1$.

(3) The infinitesimal generator of $Z(t)$, designated by $B$, has a discrete point spectrum in the half plane $\operatorname{Re} \lambda<0$ if we agree to assume that zero is not an eigenvalue of $G$.

(4) $(i \lambda)$ is an eigenvalue of $B$ if and only if $\lambda$ is a pole of the scattering matrix.

Consequently, the spectrum of B plays a central role in what follows.

It is well known from the theory of strongly continuous semigroups [4] that both $D(B)$ and $\bigcap_{n=1}^{\infty} D\left(B^{n}\right)$ are dense in $K^{\rho}$. Suppose we knew that for some time $T>0$

$$
Z(T): K^{\rho} \rightarrow D(B)
$$

Then, by the closed graph theorem, $B Z(t)$ is a bounded operator for all $t \geq T$, and a simple calculation shows that if $\lambda=\xi+i \eta \in \sigma(B)$, then $\xi<K-\log |\eta|$ for some positive constant $K$.

Now suppose, however, that for some time $T>0$

$$
Z(T): K^{\rho} \rightarrow \bigcap_{n=1}^{\infty} D\left(B^{n}\right) .
$$

Then there exists a sequence of positive real numbers $\{M(k)\}_{k=1}^{\infty}$ such that for for each $k$ and all time $t \geq T$

$$
\left\|B^{k} Z(t)\right\| \leq M(k) .
$$

Proposition 1. If $M(k)=C H^{k}(k !)^{\gamma}$, where $C$ and $H$ are positive constants, $\gamma>1$, then $\sigma(B)$ lies to the left of the curve $\xi=C_{1}-C_{2}|\eta|^{1 / \gamma}$, where $C_{1}$ and $C_{2}$ are positive constants. 
Proof. If $\lambda \in \sigma(B)$ and $M(k)=C H^{k}(k !)^{\gamma}$, then from (1.7) we may conclude that

$$
|\operatorname{Im} \lambda|^{k} e^{T \operatorname{Re} \lambda} \leq|\lambda|^{k} e^{T \operatorname{Re} \lambda} \leq C H^{k}(k !)^{\gamma} \leq C H^{k}(k)^{k \gamma},
$$

where the last inequality follows from Stirling's formula. Setting

$$
f(k)=k \log |\operatorname{Im} \lambda|+T \operatorname{Re} \lambda-\log C-k \log H-(k y) \log k,
$$

we note that $f(k)<0$ for all $k>0$. It is easily shown that $f(k)$ obtains its maximum value for $k=k_{c}$, where $\gamma \log k_{c}=\log |\operatorname{Im} \lambda|-\log H-\gamma$ and therefore

$$
0>f\left(k_{c}\right)=T \operatorname{Re} \lambda-\log C+k_{c} \gamma \geq f(k)
$$

or

$$
T \operatorname{Re} \lambda \leq \log C-(\gamma / e)(1 / H)^{1 / \gamma}|\operatorname{Im} \lambda|^{1 / \gamma} \quad \text { Q.E.D. }
$$

Since $\sigma(B)$ must lie in the left half plane, we have shown that the eigenvalues of $B$ lie in the hatched region of Figure 1 .

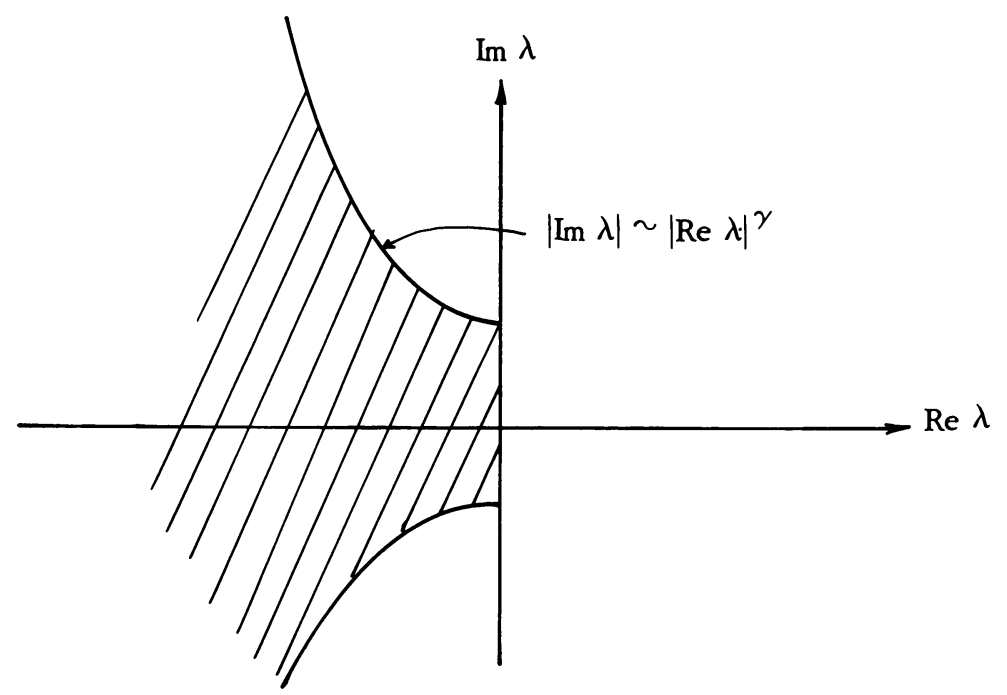

The theorems below, which will be proved in the report that follows, answer the two questions:

(I) When is condition (B2) known to be true?

(II) Assuming (B2) holds, what additional conditions are required to give us the estimate (1.7) with $M(k)=C H^{k}(k !)^{\gamma}$ ?

Theorem 3. If the bicharacteristic rays of (1.1) tend to infinity, then

(a) $Z(T)$ is compact for all $T \geq T_{0}>0$. (See [5b].)

(b) Condition (B2) is valid for some $T \geq T_{C}>0$. 
Theorem 4. If each of the coefficient matrices $A^{j}(x)$ and $B(x)$ satisfy the Gevrey condition

$$
\operatorname{Sup}_{x \in\{x:|x| \leq \rho\}}\left|D^{a} F(x)\right| \leq C H^{|a|}(|a| !)^{\mu}, \quad \mu>1,
$$

then there exist constants $C_{0}$ and $H_{0}$ such that for all time $T$ sufficiently large

$$
\left\|B^{l} Z(T)\right\| \leq C_{0} H_{0}^{l}(l !)^{3 \mu-1} \text {. }
$$

II. Question I: The proof of Theorem 3. Before proceeding to the proof of Theorem 3, we cite two useful lemmas. (For proofs, see [5b].)

Lemma 1. Let

$$
\begin{aligned}
& q_{\eta}(x) \in C_{0}^{\infty}\left(R^{k}\right) \text { sucb that } 0 \leq q_{\eta}(x) \leq 1, \\
& q_{\eta}(x)= \begin{cases}1 & \text { for }|x| \leq \eta \\
0 & \text { for }|x| \geq 2 \eta\end{cases}
\end{aligned}
$$

and define the operator $Q_{\eta}$ on $\mathcal{H}$ to be $Q_{\eta} f(x)=\left(q_{\eta}(x) I\right) f(x)$, where $I$ is the $n \times$ $n$ identity matrix. If we set $X=U(2 \rho)-V(2 \rho)$,

(i) $Q_{\rho+2 c \rho} X=X$,

(ii) $X Q_{\rho+4 c \rho}=X$, where $c=\max _{x,|\xi|=1} \tau^{1}(x, \xi)$.

Lemma 2. Using the notation of Lemma 1, for $T>4 \rho$,

$$
Z(T)=P_{+} X Q_{\rho_{+}+c \rho} U(T-4 \rho) Q_{\rho_{+} c \rho} X P_{-} .
$$

From Lemma 2, a straightforward calculation shows that

$$
B Z(T)=P_{+} X Q_{\rho_{+}+4 \rho} G U(T-4 \rho) Q_{\rho_{+} c \rho} X P_{-},
$$

and in general

$$
B^{l} Z(T)=P_{+} X Q_{\rho_{+} 4 c \rho^{\prime}} G^{l} U(T-4 \rho) Q_{\rho_{+} c \rho} X P_{-} \cdot
$$

At this point we must digress for a moment and recall the definition of the Riemann function for the equation (1.1). A distribution valued matrix $R(y, s ; x, t)$ is called the Riemann function for (1.1) if it satisfies

$$
\begin{aligned}
(\partial / \partial t-G) R(y, s ; x, t) & =0, \quad s<t, \\
R(y, s ; x, s) & =\delta(y-x) I, \\
R(y, s ; x, t) & =0, \quad s>t .
\end{aligned}
$$

Given the existence of the Riemann function, the solution to (1.1) may be then written as 


$$
u(x, t)=U(t) f(x)=\int R(y, 0 ; x, t) f(y) d y .
$$

It is known (see [1], [3b], [7]) that $R(y, 0 ; x, t)$ has its singular support along the bicharacteristic rays $\left\{x_{i}^{\lambda}(t)\right\}$ which satisfy the Hamiltonian system

$$
d x_{i}^{\lambda}(t) / d t=\tau_{\xi}^{\lambda}(x, \xi), \quad d \xi_{i}^{\lambda}(t) / d t=-\tau_{x_{i}}^{\lambda}(x, \xi)
$$

and emanate from $(y, 0)$. These rays are said to tend to infinity if for sufficiently large time, the rays lie outside any given bounded set. For the system at hand, this is equivalent to the existence of some fixed time $T_{s}$ so that any ray lying inside $|x|=a$ at time $t=0$ will fall outside $|x|=b(b>a)$ for $t>T_{s}$.

Suppose, then, that the bicharacteristic rays tend to infinity and that $f(x)$ has its support within the ball $|x|=2(\rho+2 c \rho)$. For sufficiently large time $T$, the rays all lie outside the ball $|x|=2(\rho+4 c \rho)$ and, therefore, $R(y, 0 ; x, T-4 \rho)$ is $C^{\infty}$ in $x,|x|<2(\rho+4 c \rho)$, for $|y|<2(\rho+2 c \rho)$. From (2.4) we conclude that $U(T) f(x)$ is $C^{\infty}$ for $|x| \leq 2(\rho+4 c \rho)$, and since the coefficients of $G$ are infinitely smooth, the expression on the right-hand side of (2.2) is well defined; i.e., $B^{l} Z(T)$ exists for each $l$. Note, moreover, that since $R(y, 0 ; x, T)$ is $C^{\infty},(2.1)$ and (2.4) taken together imply that $Z(T)$ is compact, since the kernel in (2.4) is $C^{\infty}$. This concludes the proof of Theorem 3 .

III. Question II: The proof of Theorem 4. Since $P_{+}$and $P_{-}$are projections and the semigroups $U(t)$ and $V(t)$ are norm preserving, it follows from (2.2) that

$$
\left\|B^{l} Z(T)\right\| \leq 4\left\|Q_{\rho+4 c \rho} G^{l} U(T-4 \rho) Q_{\rho+2 c \rho}\right\| \text {. }
$$

Lemma 3. Let $\alpha=\rho+4 c \rho, \beta=\rho+2 c \rho$. Then

$$
\left\|B^{l} Z(T)\right\| \leq 4\left(\int_{|x| \leq 2 \alpha} d x \int_{|y| \leq 2 \beta} d y\left|G^{l} R(y, 0 ; x, T-4 \rho)\right|^{2}\right)^{1 / 2} .
$$

Proof. Let $g(y) \in \mathcal{H}$ and $g(y)^{\#}=Q_{\beta} g(y)$. Then

$$
U(T-4 \rho) Q_{\beta} g(y)=\int_{|y| \leq 2 \beta} R(y, 0 ; x, T-4 \rho) g(y)^{\#} d y
$$

and

$$
\begin{aligned}
\left\|Q_{a} G^{l} U(T-4 \rho) Q_{\beta} g\right\|^{2} & \leq \int_{|x| \leq 2 a} d x\left|\int_{|y| \leq 2 \beta} G^{l} R(y, 0 ; x, T-4 \rho) g(y)^{\#} d y\right|^{2} \\
& \leq \int_{|x| \leq 2 a} d x\left\{\int_{|y| \leq 2 \beta}\left|G^{l} R(y, 0 ; x, T-4 \rho) \| g(y)^{\#}\right| d y\right\}^{2} \\
& \leq \int_{|x| \leq 2 a} d x\left(\int_{|y| \leq 2 \beta} d y\left|G^{l} R(y, 0 ; x, T-4 \rho)\right|^{2} d y\right)\|g\|^{2} .
\end{aligned}
$$

Since $g(y)$ is arbitrary, if follows that 


$$
\left\|Q_{\alpha} G^{l} U(T-4 \rho) Q_{\beta}\right\| \leq\left(\int_{|x| \leq 2 \alpha} d x \int_{|y| \leq 2 \beta} d y\left|G^{l} R(y, 0 ; x, T-4 \rho)\right|^{2}\right)^{1 / 2}
$$

and, in light of (3.1), the lemma is proved.

We have reduced our problem, therefore, to obtaining estimates on the Riemann function. The type of estimates that we desire were originally produced by A. Friedman [2], and more recently extended by the present author [3a, b]. To summarize these results we first need the following definitions [6].

Let $\{H(q)\}_{q=1}^{\infty}$ be a montone increasing sequence of positive real numbers satisfying

(a) $H(1) \geq 1$.

(b) For all integers $p, q, 0 \leq p<q<\infty,(\underset{p}{q}) H(p) H(q-p) \leq A H(q)$ for some constant $A \geq 1$; we define $H(0)=A$.

(c) For all $q \geq 1$, there exist constants $\beta$ and $K_{0}$ such that $(H(q+1) / H(q))$ $<K_{0} \beta^{q}$.

(d) $\sum_{q=1}^{\infty}(H(q) / H(q+1))<\infty$.

If $\Omega$ is an open set in $R^{k}, C(H(q), \Omega)$ will denote the subset of $C_{0}^{\infty}(\Omega)$ in which

$$
\sup _{x \in \boldsymbol{\Omega}}\left|D^{\alpha} f(x)\right| \leq C K^{|\alpha|} H(|\alpha|),
$$

where the constants $C$ and $K$ depend only on the choice of function $f(x) \in C_{0}^{\infty}(\Omega)$. The classical example of such functions are the Gevrey functions of type $\mu>1$ which correspond to the sequence $H(q)=(q !)^{\mu}$.

Let $V$ be any open, bounded set of points in $(x, t)$ whose closure does not intersect the characteristic conoid emanating from $(y, 0)$. Set $V^{\sigma}=V \cap\{t: 0 \leq t$ $\leq \sigma\}$ and denote by $W_{K}^{\sigma}$ the cylinder $\{(x, t): 0 \leq t \leq \sigma,|x| \leq K\}$. For $R_{0}$ such that $\{$ closure $V\} \cap\{t=0\} \subset\left\{|x|<R_{0}\right\}$, choose $R, 0<R<R_{0}$, so that the domain of dependence of $w_{R}^{\epsilon}$ is contained within the domain of dependence of $W_{R_{0}}^{\epsilon_{0}}$ and such that $V^{\epsilon_{0}} \subset W_{R}^{\epsilon_{0}^{R}}$ for some $\epsilon_{0}>0$. Then we have

Theorem 5. (See [2], [3b].) If the coefficient matrices $A^{j}(x), B(x)$ of (1.1) belong to $C\left(H(q), W_{R_{0}}^{\epsilon_{0}}\right)$, then the Riemann function $R(y, 0 ; x, t)$ belongs to $C\left(\widetilde{H}(q), V^{\epsilon_{0}}\right)$ with $\widetilde{H}(q) \leq H(3 q) / q$ !.

We introduce two notational conveniences. For $g(x, t) \in L_{2}\left(R^{k} \times[0, \infty)\right)$

$$
\begin{aligned}
& \|g(\cdot, t)\|_{(R)}^{2}=\int_{|x| \leq R}|g(x, t)|^{2} d x, \\
& n\|g(\cdot, t)\|_{(R)}^{2}=\sum_{|a|=n}\left\|D^{a} g(x, t)\right\|_{(R)}^{2} .
\end{aligned}
$$


Theorem 6. If $f(x, t) \in C^{\infty}\left(R^{k} \times[0, t]\right)$ and satisfies

$$
{ }_{n}\|f(\cdot, t)\|_{(R)} \leq C K^{n} H(n),
$$

then under the bypotheses of Theorem 5

$$
\left\|G^{n} f(\cdot, t)\right\|_{(R)} \leq \widetilde{C} K^{n} H(n) .
$$

If we temporarily assume this result, it follows from Theorem 5 , that if the rays tend to infinity, then for sufficiently large time $T$, the rays lie outside $|x|$ $=2 \alpha$ and

$$
\left\|G^{n} R(y, 0 ; x, T-4 \rho)\right\|_{(2 a)} \leq C K^{n} \tilde{H}(n)
$$

provided $A^{j}(x), B(x) \in C(H(q),|x|<\rho+\delta)$. Combining this estimate with (3.1), the proof of Theorem 4 is now complete. It remains to verify Theorem 6 .

Proof. If we agree to retain only the order of derivatives which appear in the following calculations, then by Leibniz's formula, for $|\alpha|=n$

$$
\begin{aligned}
& D^{a}(G f)=\sum_{j=1}^{k} \sum_{l=0}^{n}\left(\begin{array}{l}
n \\
l
\end{array}\right)\left(D^{l} A^{j}(x)\right)\left(D^{n-l+1} f\right)+\sum_{l=0}^{n}\left(\begin{array}{l}
n \\
l
\end{array}\right)\left(D^{l} B\right)\left(D^{n-l} f\right) \\
& \quad \Rightarrow\left\|D^{a}(G f)\right\|_{(R)} \leq \sum_{l=0}^{n}\left(\begin{array}{l}
n \\
l
\end{array}\right)\left[\sum_{j=1}^{k}\left\|D^{l} A^{j}\right\|_{(R)}\left\|D^{n-l+1} f\right\|_{(R)}+\left\|D^{l} B\right\|_{(R)}\left\|D^{n-l} f\right\|_{(R)}\right] .
\end{aligned}
$$

Using Hölder's inequality

$$
\begin{array}{r}
\left\|D^{a} G\right\|_{(R)}^{2} \leq\left[\sum _ { l = 0 } ^ { n } ( \begin{array} { l } 
{ n } \\
{ l }
\end{array} ) \left\{\left(\sum_{j=1}^{k}\left\|D^{l} A^{i}\right\|_{(R)}^{2}\right)^{1 / 2}\left(\sum_{j=1}^{k}\left\|D^{n-l+1} f\right\|_{(R)}^{2}\right)^{1 / 2}\right.\right. \\
\left.\left.+\left\|D^{l} B\right\|_{(R)}\left\|D^{n-l} f\right\|_{(R)}\right\}\right]^{2} \\
\Rightarrow{ }_{n}\|G f\|_{(R)}^{2} \leq\left(\sum _ { | a | = n } \left[\sum _ { l = 0 } ^ { n } ( \begin{array} { l } 
{ n } \\
{ l }
\end{array} ) \left\{\left(\sum_{j=1}^{k}\left\|D^{l} A^{j}\right\|_{(R)}^{2}\right)^{1 / 2}\left(\sum_{j=1}^{k}\left\|D^{n-l+1} f\right\|_{(R)}^{2}\right)^{1 / 2}\right.\right.\right. \\
\left.\left.\left.+\left\|D^{l} B\right\|_{(R)}\left\|D^{n-l} f\right\|_{(R)}\right\}\right]\right)^{2} .
\end{array}
$$

If we set $C(l)^{2}=\sum_{j=1}^{k} l\left\|A^{j}\right\|_{(R)}^{2}$, then

$$
{ }_{n}\|G f\|_{(R)} \leq \sum_{l=0}^{n}\left(\begin{array}{l}
n \\
l
\end{array}\right)\left[C(l)_{n-l+1}\|f\|_{(R)}+{ }_{l}\|B\|_{(R) n-l}\|f\|_{(R)}\right] .
$$

Using the estimate (3.5) and the similar estimates for $C(l)$ and $\|B\|$ in (3.8), we obtain the estimate 


$$
n\|G\|_{(R)} \leq \tilde{C} \tilde{K}^{n} W H(n+1)
$$

where $\widetilde{C}, \widetilde{K}$ and $W$ depend only on the constants appearing in the other estimates. We now make an induction hypothesis; i.e., for all $n$ we assume

$$
{ }_{n}\left\|G^{k} f\right\|_{(R)} \leq \widetilde{C} K^{n+k} W^{k} H(n+k), \quad k=1,2, \ldots, m .
$$

Then, using (3.8)

$$
\begin{aligned}
{ }_{n}\left\|G^{m+1} f\right\|_{(R)} & \leq \sum_{l=0}^{n}\left(\begin{array}{l}
n \\
l
\end{array}\right)\left[C(l)_{n-l+1}\left\|G^{m} f\right\|_{(R)}+{ }_{l}\|B\|_{(R) n-l}\left\|G^{m} f\right\|_{(R)}\right] \\
& \leq \widetilde{C} K^{m+n+1} W^{m+1} M(n+m+1) .
\end{aligned}
$$

This verifies the induction hypothes is for $k=m+1$, and if we now set $n=0$, we have $\left\|G^{m+1} f\right\|_{(R)} \leq \widetilde{C}(\widetilde{K} W)^{m+1} H(m+1)$, thereby completing the proof.

Corollary 1. If $A^{j}(x), B(x) \in C\left((q !)^{\mu}, \Omega\right)$, with $\Omega \supset\{x:|x| \leq \rho\}$ and $\mu>1$, then the scattering matrix $\mathcal{S}(z)$ associated with the system (1.1) is meromorphic in the complex plane, with all the poles lying in the upper half plane above the curve

$$
\operatorname{Im} \sigma=C_{1}-C_{2}|\operatorname{Re} \sigma|^{1 /(3 \mu-1)}
$$

with $C_{1}$ and $C_{2}$ positive constants.

Proof. The result follows immediately from Theorems 2 and 4 .

Remark. Lax and Phillips have shown [5c] that the ir techniques for scattering theory are applicable to the acoustic equation

$$
\begin{aligned}
u_{t t}(x, t) & =\Delta u(x, t)-q(x) u(x, t), \quad t \geq 0, x \in R^{k}, \\
u(x, 0) & =f(x), \quad u_{t}(x, 0)=g(x),
\end{aligned}
$$

where $q(x) \in L_{2}\left(R^{k}\right)$ and is assumed to have compact support. The bicharacteristics for this equation are straight lines and consequently condition (B2) is known to hold. Moreover, if it is assumed that $q(x) \in C\left((k !)^{\mu}\right.$, supp $\left.(q(x))\right)$, the following result may be proved in a manner analogous to the above.

Theorem 7. [3a]. If $\mathcal{S}(z)$ is the Heisenberg scattering matrix associated with (3.10), then $\mathfrak{S}(z)$ is a meromorphic function of $z$, with a finite number of poles in the lower half plane on the imaginary axis and poles in the upper half plane above the curve

$$
\operatorname{Im} \sigma=C_{1}-C_{2}|\operatorname{Re} \sigma|^{1 /(3 \mu-1)}, \quad C_{1}, C_{2}>0 .
$$




\section{BIBLIOGRAPHY}

1. R. Courant and P. D. Lax, The propagation of discontinuities in wave motion, Proc. Nat. Acad. Sci. U. S. A. 42 (1956), 872-876. MR 18, 399.

2. A. Friedman, Regularity of fundamental solutions of hyperbolic equations, Arch. Rational Mech. Anal. 11 (1962), 62-96. MR $26 \# 1624$.

3a. W. L. Goodhue, On the location of the poles of the scattering matrix for symmetric hyperbolic equations, Thesis, New York University, October 1971.

3b. On the regularity of the Riemann function for hyperbolic equations, Trans. Amer. Math. Soc. 175 (1973), 483-490.

4. E. Hille and R. S. Phillips, Functional analysis and semi-groups, rev. ed., Amer. Math. Soc. Colloq. Publ., vol. 31, Amer. Math. Soc., Providence, R. I., 1957. MR 19, 664.

5a. P. D. Lax and R. S. Phillips, Scattering theory, Pure and Appl. Math., vol. 26, Academic Press, New York, 1967. MR $36 \# 530$.

5b. Lecture notes on scattering theory, Rocky Mountain Regional Summer Math. Seminar, Flagstaff, Arizona, 1969.

5c. The acoustic equation with an indefinite energy form and the Schrödinger equation, J. Functional Analysis 1 (1967), 37-83. MR 36 \#531.

6. J. L. Lions and E. Magenes, Probièmes aux limites non homogènes et applications. Vol. 3, Dunod, Paris, 1970.

7. D. Ludwig, Exact and asymptotic solutions of the Cauchy problem, Report NYO2545, Atomic Energy Commission Research and Development. 46556

DEPARTMENT OF MATHEMATICS, UNIVERSITY OF NOTRE DAME, NOTRE DAME, INDIANA 